\title{
Ansiedade-traço em estudantes universitários de Aracaju (SE)
}

\author{
Trait anxiety in Brazilian university students from Aracaju
}

\author{
Marcel Magalhães Alves Gama ${ }^{1}$, Gustavo Souza Moura ${ }^{1}$, Ronaldo Franca Araújo ${ }^{1}$, Flavia Teixeira- \\ Silva ${ }^{2}$
}

${ }^{1}$ Acadêmico de Medicina, Universidade Federal de Sergipe (UFS), São Cristóvão, SE. ${ }^{2}$ Doutora. Professora adjunta, UFS.

\begin{abstract}
Resumo
Introdução: No estudo da ansiedade, dois conceitos distintos são encontrados: ansiedade-estado, que se refere a um estado emocional transitório caracterizado por sentimentos subjetivos de tensão que podem variar em intensidade ao longo do tempo; e ansiedadetraço, a qual se refere a uma disposição pessoal, relativamente estável, a responder com ansiedade a situações estressantes e a uma tendência a perceber um maior número de situações como ameaçadoras. Na literatura, encontram-se várias escalas com o objetivo de avaliar esses dois aspectos, mas o Inventário de Ansiedade Traço-Estado, de Spielberger et al. (1970), é o mais utilizado. Os objetivos do presente estudo foram: 1) descrever os escores do Inventário de Ansiedade Traço-Estado em uma amostra de estudantes universitários de Aracaju (SE), onde as pressões da vida moderna estão menos presentes; e 2) compará-los com os resultados obtidos em São Paulo (SP).

Método: Foram avaliados 498 estudantes universitários (53,6\% do sexo feminino), sendo que 87,6\% tinham menos que 30 anos, $89,6 \%$ eram solteiros, e $24,7 \%$ tinham uma ocupação profissional.

Resultados: Os dados do presente estudo mostraram que, em Aracaju, os maiores níveis de ansiedade estão entre mulheres, solteiros e aqueles com até 30 anos de idade. Na comparação entre Aracaju e São Paulo, foi encontrado menor nível de ansiedade-traço nos estudantes aracajuanos, sendo que essa diferença parece residir principalmente no grupo de indivíduos de até 30 anos de idade.

Conclusão: Os resultados aqui apresentados sugerem que a ansiedade-traço dos estudantes universitários de Aracaju é menor que a dos estudantes universitários de São Paulo, apoiando a hipótese de que grandes cidades possam favorecer o desenvolvimento da ansiedade.
\end{abstract}

Descritores: Ansiedade, personalidade, estudantes, cidades.

\begin{abstract}
Introduction: Two different concepts are found in the study of anxiety: state anxiety, which is a transient state characterized by subjective feelings of tension that can vary in intensity with time; and trait anxiety, which is a relatively stable personality disposition to respond to a wider range of situations as dangerous or threatening. In the literature, it is possible to find various scales that aim at evaluating these two aspects of anxiety, although the State-Trait Anxiety Inventory by Spielberger et al. (1970) is the most widely used. The objectives of the present study were: 1) to describe the State-Trait Anxiety Inventory scores of a sample of university students from Aracaju, where modern life stressors are not as present as they are in the large Brazilian cities such as São Paulo, and 2) to compare the results obtained to those from a previous study performed in São Paulo.

Method: Four hundred and ninety-eight university students (53.6\% female) from Aracaju were evaluated: $87.6 \%$ were under 30 years of age, $89.6 \%$ were single and $24.7 \%$ had a job.

Results: The data obtained from this study showed that in Aracaju the highest levels of anxiety were among individuals who were either single, under 30 or female. The comparison between Aracaju and São Paulo found that the Aracajunian students had lower levels of anxiety, and this difference seemed to reside mainly in the group under 30 years of age.

Conclusion: The results presented here suggest that trait anxiety in university students from Aracaju is lower than in university students from São Paulo, supporting the hypothesis that life in large cities can favor the development of anxiety.

Keywords: Anxiety, personality, students, cities.
\end{abstract}

\section{Correspondência:}

Flavia Teixeira-Silva, Universidade Federal de Sergipe, Departamento de Fisiologia, Av. Marechal Rondon, s/nº, Jardim Rosa Elze, CEP 49100-000, São Cristóvão, SE.

Copyright (C) Revista de Psiquiatria do Rio Grande do Sul - APRS

Recebido em 10/09/2007. Aceito em 21/11/2007. 


\section{Introdução}

A ansiedade, apesar de ser um fenômeno universal, vivenciado por todo ser humano, inúmeras vezes ao longo de sua vida, ainda não possui uma definição exata. Existe pouca concordância entre o que os vários estudiosos chamam de ansiedade ${ }^{1}$, chegando mesmo a ser considerada como irredutível e indefinível ${ }^{2}$. Embora ainda sem uma definição exata, a ansiedade pode ser facilmente notada. No homem, ela é percebida como uma emoção caracterizada por um alerta tenso e fisicamente exaustivo, focalizado em um perigo ou emergência iminente e inevitável, embora não objetivamente aparente, com uma incerteza dolorosa sobre a possibilidade de se resolver a situação ${ }^{3}$.

No estudo da ansiedade, encontramos dois conceitos distintos: a ansiedade-estado, referente a um estado emocional transitório, caracterizado por sentimentos subjetivos de tensão que podem variar em intensidade ao longo do tempo, e a ansiedadetraço, a qual se refere a uma disposição pessoal, relativamente estável, a responder com ansiedade a situações estressantes e uma tendência a perceber um maior número de situações como ameaçadoras ${ }^{4}$.

Na literatura, encontram-se várias escalas com o objetivo de avaliar esses dois aspectos, mas sem dúvida o Inventário de Ansiedade Traço-Estado (IDATE), de Spielberger et al., é o mais utilizado 5 . Esse inventário é constituído de 40 afirmações, a respeito dos sentimentos do sujeito, distribuídas em duas partes. A primeira parte avalia a ansiedadeestado, enquanto a segunda avalia o traço ansioso. Cada parte consiste de 20 afirmações descritivas de sentimentos pessoais, os quais os sujeitos graduam em relação à intensidade com que estão ocorrendo naquele momento (parte I) ou em relação à freqüência com que ocorrem geralmente (parte II), através de uma escala que varia de 1 a 4 pontos. O IDATE foi traduzido e validado para o nosso meio por Biaggio \& Natalício em $1979^{6}$ e, desde então, vem sendo revalidado em outros estudos ${ }^{7,8}$. Nestes estudos, foram obtidos escores médios de ansiedade-traço da população brasileira, os quais têm servido de base para investigações em que são selecionados sujeitos de acordo com seu nível de ansiedade-traço-12. Contudo, os estudos de validação do IDATE utilizaram, em suas amostras, somente voluntários residentes nas cidades de São Paulo ou Rio de Janeiro, sudeste do Brasil. Sendo o Brasil um país de dimensões continentais, que possui diferentes culturas regionais ${ }^{13}$, é bem possível que os valores médios de ansiedade-traço difiram entre as diversas regiões do Estado brasileiro. Além disso, São Paulo e Rio de
Janeiro são grandes metrópoles, onde milhões de pessoas competem por um espaço num mercado de trabalho cada vez mais industrializado ${ }^{14}$. Considerando que muitos acreditam que as pressões de uma sociedade moderna, tecnológica e competitiva sejam fatores de grande importância no desenvolvimento da ansiedade ${ }^{15}$, não seria surpreendente se a população das cidades estudadas apresentasse valores médios de ansiedade-traço mais altos em comparação com populações de outras cidades brasileiras. Dessa forma, seria interessante que estudos semelhantes aos já realizados no sudeste do país fossem realizados em outras regiões do Brasil, a fim de se conhecer possíveis variabilidades interculturais em nosso país, no que diz respeito ao traço ansioso.

Assim sendo, os objetivos do presente estudo foram: 1) descrever os escores do IDATE-traço para uma amostra de estudantes universitários de Aracaju (SE), uma cidade do nordeste brasileiro, onde as pressões da vida moderna estão menos presentes; e 2) compará-los a resultados obtidos no mais recente estudo realizado em São Paulo ${ }^{8}$.

\section{Métodos}

\section{Sujeitos e procedimento}

Foram avaliados 498 estudantes universitários (231 homens, 267 mulheres) de diversos cursos da Universidade Federal de Sergipe. Foi solicitado aos estudantes, em sala de aula, que respondessem voluntariamente a uma versão do IDATE traduzida e validada para o nosso meio por Biaggio \& Natalício ${ }^{6}$.

Antes da aplicação do inventário, foi dito aos sujeitos que se tratava de uma pesquisa para avaliar características de personalidade e que os indivíduos que escolhessem participar poderiam identificar-se apenas por suas iniciais. Foi enfatizado também que os pesquisadores envolvidos no estudo comprometerse-iam a utilizar os dados e o material coletado somente para a pesquisa, analisando as informações obtidas em conjunto com as de outros voluntários, sem que fosse divulgada a identificação de nenhum participante.

\section{Ética}

Previamente ao seu desenvolvimento, o presente estudo foi aprovado pelo Comitê de Ética em Pesquisa com Seres Humanos da Universidade Federal de Sergipe. 


\section{Instrumento}

Foi utilizada somente a parte II do IDATE, que avalia a tendência do sujeito a responder de forma ansiosa às situações de vida (ansiedade-traço). Essa parte consiste de 20 afirmações descritivas de sentimentos pessoais, com escore total variando entre 20 e 80 pontos, sendo que os valores mais altos indicam maiores níveis de ansiedade.

\section{Análises estatísticas}

As comparações por características demográficas (idade, sexo, estado civil e ocupação profissional) foram feitas por ANOVA de uma via, seguida de teste a posteriori de Tukey quando apropriado. Em relação à idade, os dados foram agrupados em três categorias: A (idade menor ou igual a 30 anos), B (idade entre 31 e 45 anos) e C (idade superior a 45 anos).

Já as comparações entre os dados obtidos neste estudo e dados de um estudo realizado em São Paulo foram feitas utilizando-se teste $t$ de Student.

\section{Resultados}

Os dados obtidos dos estudantes de Aracaju foram agrupados de acordo com características sociodemográficas (Tabela 1). No presente estudo, as diferenças entre os gêneros foram significativas, com mulheres apresentando maiores escores de ansiedade que os homens $\left(F_{1,468}=15,65 ; p=0,00009\right)$. Também foram significativas as diferenças de acordo com o estado civil, com os solteiros apresentando os maiores escores $\left(\mathrm{F}_{1,461}=5,68 ; \mathrm{p}=0,018\right)$. Em relação à ocupação profissional, não houve diferença significativa entre os estudantes que trabalhavam e os que não trabalhavam $\left(\mathrm{F}_{1,466}=0,26 ; \mathrm{p}=0,61\right)$.

Com relação à faixa etária, quando as três categorias (A, B e C) foram analisadas, a ANOVA revelou uma diferença significativa entre os grupos $\left(\mathrm{F}_{2,478}=4,12 ; \mathrm{p}=0,017\right)$, mas essa diferença não foi confirmada pelo teste de Tukey. Isso pode estar relacionado ao fato de a categoria $\mathrm{C}$ ter um número muito pequeno de representantes, o que diminui o poder do teste a posteriori. Dessa forma, quando

Tabela 1 - Escores do IDATE-traço distribuídos segundo características sociodemográficas dos estudantes universitários de Aracaju (SE)

\begin{tabular}{lccc}
\hline Características sociodemográficas & $\mathbf{n}$ & $\mathbf{\%}$ & $\begin{array}{c}\text { IDATE-traço } \\
\text { Média (DP) }\end{array}$ \\
\hline Idade & & & \\
$\quad \leq 30$ & 436 & 87,6 & $41,8(9,4)$ \\
$31-45$ & 39 & 7,8 & $37,5(7,5)$ \\
$\quad>45$ & 11 & 2,2 & $39,4(8,5)$ \\
$\quad$ NI & 12 & 2,4 & $40,4(7,4)$ \\
Estado civil & & & \\
$\quad$ Solteiro & 446 & 89,6 & $41,8(9,3)$ \\
$\quad$ Casado & 45 & 9,0 & $38,2(8,7)$ \\
$\quad$ NI & 7 & 1,4 & $39,3(7,6)$ \\
Sexo & & & \\
$\quad$ Feminino & 267 & 53,6 & $42,9(9,1)$ \\
$\quad$ Masculino & 231 & 46,4 & $39,6(9,2)$ \\
Ocupação profissional & & & \\
$\quad$ Sim & 123 & 24,7 & $41,0(9,7)$ \\
$\quad$ Não & 372 & 74,7 & $41,5(9,1)$ \\
NI & 4 & 0,8 & $39,5(12,0)$ \\
Total & 498 & 100,0 & $41,4(9,3)$ \\
\hline
\end{tabular}

$\mathrm{DP}=$ desvio padrão; IDATE $=$ Inventário de Ansiedade Traço-Estado; NI = não identificado. 
analisadas somente as categorias A e B, encontramos que os indivíduos com até 30 anos apresentam escores mais altos quando comparados aos que possuem idade entre 31 e 45 anos $\left(F_{2,468}=7,68 ; \mathrm{p}=\right.$ 0,006).

Na comparação dos dados do presente estudo com os obtidos em São Paulo, num estudo que avaliou 1.080 universitários $^{8}$, foram encontradas as diferenças significativas mostradas na Tabela 2. Índices significativamente menores de ansiedade foram encontrados nos indivíduos aracajuanos pertencentes aos seguintes grupos: faixa etária $\mathrm{B}$, solteiros, homens e indivíduos com ocupação profissional. Independentemente de idade, estado civil, gênero e ocupação profissional, os estudantes universitários de Aracaju apresentaram menores escores do IDATE quando comparados aos estudantes universitários de São Paulo.

\section{Discussão}

O objetivo do presente estudo foi descrever os escores do IDATE-traço para uma amostra de estudantes universitários de Aracaju e compará-los a resultados obtidos em São Paulo. Em ambos os estudos, os maiores escores de ansiedade foram encontrados em mulheres, solteiros e indivíduos com até 30 anos. Este achado está de acordo com outros estudos envolvendo o IDATE ${ }^{16,17}$.

Estudos epidemiológicos têm mostrado escores de ansiedade maiores em amostras mais jovens comparadas às mais velhas ${ }^{18}$.

As diferenças entre os gêneros, com mulheres apresentando escores maiores do que os homens, também estão em concordância com outros estudos ${ }^{19-23}$. De acordo com pesquisas da comunidade científica americana, mulheres têm mais predisposição do que homens para desenvolver transtorno do pânico (7,7 versus $2,9 \%$ ), transtorno obsessivo compulsivo (6,6 versus $3,6 \%)$ ou transtorno de estresse pós-traumático (12,5 versus $6,2 \%$ ) durante a vida ${ }^{24}$. É possível que essa maior predisposição esteja associada ao fato de que mulheres apresentem naturalmente um traço mais ansioso que os homens.

Quanto aos indivíduos mais jovens, não há dados científicos que justifiquem os menores escores de ansiedade-traço em universitários menores que 30 anos residentes em Aracaju em comparação aos de São Paulo. Todavia, pode-se inferir que os residentes em São Paulo estão expostos a um número infindável de estímulos que conduzem ao estresse, modulando, assim, a ansiedade-traço. Para boa parte da população dos grandes centros urbanos, em especial dos países pobres, viver em metrópoles passou a ser sinônimo de

Tabela 2 - Comparação entre os níveis de ansiedade-traço de estudantes universitários de Aracaju $(\mathrm{SE})$ e de São Paulo (SP) ${ }^{8}$

\begin{tabular}{|c|c|c|c|}
\hline Características sociodemográficas & $\begin{array}{l}\text { Aracaju (SE) } \\
\text { Média (DP) }\end{array}$ & $\begin{array}{l}\text { São Paulo (SP) } \\
\text { Média (DP) }\end{array}$ & $\begin{array}{c}\text { Teste } \boldsymbol{t} \text { de Student } \\
\text { (p) }\end{array}$ \\
\hline \multicolumn{4}{|l|}{ Idade } \\
\hline$\leq 30$ & $41,8(9,4)$ & $43,8(10,7)$ & 0,0008 \\
\hline $31-45$ & $37,5(7,5)$ & $40,2(11,5)$ & 0,1715 \\
\hline$>45$ & $39,4(8,5)$ & $37,5(9,7)$ & 0,6042 \\
\hline \multicolumn{4}{|l|}{ Estado civil } \\
\hline Solteiro & $41,8(9,3)$ & $43,8(10,8)$ & 0,0008 \\
\hline Casado & $38,2(8,7)$ & $40,1(10,7)$ & 0,2801 \\
\hline \multicolumn{4}{|l|}{ Sexo } \\
\hline Feminino & $42,9(9,1)$ & $43,7(10,7)$ & 0,2706 \\
\hline Masculino & $39,6(9,2)$ & $41,6(11,5)$ & 0,0389 \\
\hline \multicolumn{4}{|l|}{ Ocupação profissional } \\
\hline Sim & $41,0(9,7)$ & $43,3(11,0)$ & 0,0287 \\
\hline Não & $41,5(9,1)$ & $42,9(10,7)$ & 0,5204 \\
\hline Total & $41,4(9,3)$ & $43,4(10,8)$ & 0,0004 \\
\hline
\end{tabular}

$\mathrm{DP}=$ desvio padrão. 
desemprego, miséria, violência, congestionamento, favelas e poluição ${ }^{25,26}$. É possível que, para um jovem que ainda vai ingressar no mercado de trabalho, essas estatísticas sejam ameaçadoras.

$\mathrm{E}$, finalmente, os aracajuanos que dividem sua jornada entre trabalho e estudo parecem se preocupar menos com isso que os universitários/trabalhadores paulistanos, talvez também um reflexo da maior competitividade presente nos grandes centros.

De uma forma ou de outra, os escores de ansiedade-traço de estudantes universitários de Aracaju são significativamente menores que os de estudantes universitários de São Paulo, o que apóia a hipótese de que grandes cidades possam favorecer o desenvolvimento da ansiedade. Não se pode ignorar, no entanto, as diferenças culturais entre as duas cidades, que poderiam também contribuir para os diferentes níveis de ansiedade dos estudantes, independentemente da presença das tensões metropolitanas. Uma maneira de evitar essas influências culturais seria avaliar os índices de ansiedade-traço em grandes e pequenas cidades de uma mesma região do país. Enquanto isso não é feito, o presente estudo deixa claro que habitantes de diferentes regiões do Brasil possuem diferentes escores de IDATE-traço. Tais diferenças, embora possam não ser clinicamente relevantes, certamente mudariam a classificação de indivíduos em termos de níveis de ansiedade nos estudos científicos. Considerando-se o padrão usual de classificação em alta ansiedade ( $<$ média -1 desvio padrão), média ansiedade (média \pm 1 desvio padrão) e baixa ansiedade ( $>$ média +1 desvio padrão), um universitário aracajuano com IDATE-traço de 52 pontos seria classificado como altamente ansioso, mas seu nível de ansiedade seria considerado apenas mediano se ele morasse em São Paulo. Sendo assim, torna-se clara a importância de cada região do Brasil possuir a descrição de seus próprios escores de ansiedade.

\section{Conclusão}

Os dados do presente estudo mostram maiores níveis de ansiedade entre mulheres, solteiros e aqueles com até 30 anos de idade.

Em comparação com os resultados obtidos em São Paulo, os estudantes universitários aracajuanos apresentam menores escores de ansiedade-traço que os universitários paulistanos, sugerindo que dados encontrados numa dada região do país não possam ser extrapolados para todo o Brasil.

\section{Referências}

1. Pessotti I. Ansiedade. São Paulo: Pedagógica e Universitária; 1978.

2. Lader M. The nature of anxiety. Brit J Psychiat. 1972;121(564):481-91.

3. Martin P. Animal models sensitive to antianxiety agents. Acta Psychiatr Scand Suppl. 1998;98(393):74-80.

4. Spielberger CD, Gorsuch RL, Lushene RE. Manual for the statetrait anxiety inventory ("self-evaluatin questionaire"). California: Consulting-Psychologists; 1970.

5. Andreatini R, Seabra ML. A estabilidade do IDATE-traço: avaliação após cinco anos. Rev ABP-APAL; 1993;15(1):21-5.

6. Biaggio AMB, Natalício L. Manual para o Inventário de Ansiedade TraçoEstado (IDATE). Rio de Janeiro: CEPA; 1979.

7. Gorenstein C, Andrade L. Validation of a portuguese version of the beck depression inventory and the state-trait anxiety inventory in Brazilian subjects. Braz J Med Biol Res. 1996;29(4):453-7.

8. Andrade L, Gorenstein C, Vieira Filho AH, Tung TC, Artes R. Psychometric properties of the Portuguese version of the StateTrait Anxiety Inventory applied to college students: factor analysis and relation to the Beck Depression Inventory. Braz J Med Biol Res. 2001;34(3):367-74.

9. Gorenstein C, Pompéia S, Andrade L. Scores of brazilian university students on the beck depression and the state-trait anxiety inventories. Psychological Reports. 1995;77(2):635-41.

10. Leite JR, Seabra MLV, Sartori VA, Andreatini R. The VideoRecorded Stroop ColorWord Test as a new model of experimentally-induced anxiety. Prog Neuropsychopharmacol \& Biol Psychiat. 1999;23:809-22.

11. Silva FT, Leite JR. Physiological modifications and increase in state anxiety in volunteers submitted to the Stroop Color-Word Interference Test: a preliminary study. Physiol Behav. 2000;70(1-2):113-8.

12. Teixeira-Silva F, Prado GB, Ribeiro LC, Leite JR. The anxiogenic vídeo-recorded Stroop Color-Word Test: psychological and physiological alterations and effects of diazepam. Physiol Behav. 2004;82(2-3):215-30.

13. Coelho MA. Regionalização e planejamento regional do Brasil. In: Geografia do Brasil. Coelho MA. São Paulo: Moderna; 1996. p.

14. Instituto Brasileiro de Geografia e Estatística. Cidades@: o Brasil município por município. Disponível em: $<$ http:// www.ibge.gov.br/cidadesat/default.php>. Acesso em: 02 set. 2007.

15. Fisher S. The causes and control of anxiety. Br J Hosp Med. 1990;44(3):194-7.

16. Nakazato K, Shimonaka Y. The Japanese State-Trait Anxiety Inventory: age and sex differences. Percept Mot Skills. 1989;69(2):611-7.

17. Stanley MA, Beck JG, Zebb BJ. Psychometric properties of four anxiety measures in older adults. Behav Res Ther. 1996;34(10):827-38.

18. Regier DA, Boyd JH, Burke JD Jr., Rae DS, Myers JK, Kramer $\mathrm{M}$, et al. One-month prevalence of mental disorders in the United States. Based on five Epidemiologic Catchment Area Sites. Arch Gen Psychiatry. 1988;45(11):977-86.

19. Guida FW, Ludlow LH. A cross-cultural study of test anxiety. J Cross-Cultural Psychology. 1989;20:178-90.

20. Inderbitzen HM, Hope DA. Relationship among adolescent reports of social anxiety, anxiety, and depressive simptoms. J Anx Disord. 1995;9:385-96.

21. Pajares F, Kranzler J. Self-efficacy beliefs and general mental ability in mathematical problem-solving. Contemporary Educational Psychology. 1995;20:426-43.

22. Silverman WK, La Greca AM, Wasserstein S. What do children worry about? Worries and their relation to anxiety. Child Dev. 1995;66(3):671-86. 
23. Steer RA, Beck AT, Beck JS. Sex effect sizes of the Beck Anxiety Inventory for psychiatric outpatients matched by age and principal disorders. Assessment. 1995;2:31-8.

24. Kessler RC, McGonagle KA, Zhao S, Nelson CB, Hughes M, Eshleman S. Lifetime and 12-month prevalence of DSM-III-R psychiatric disorders in the United States. Results from the National Comorbidity Survey. Arch Gen Psychiatry. 1994;51(1):8-19.
25. Bueno, LMM. Projeto e favela: metodologia para projetos de urbanização. [tese]. São Paulo: Faculdade de Arquitetura e Urbanismo da Universidade de São Paulo; 2000.

26. Clichevski N. Informalidad y segregación urbana en América Latina: una aproximación. Santiago do Chile: CEPAL/ECLAC; 2000 . 